\title{
MiKHAIL BOROVOI The defect of weak approximation for homogeneous spaces
}

Annales de la faculté des sciences de Toulouse $6^{e}$ série, tome $8, \mathrm{n}^{\circ} 2$ (1999), p. 219-233

<http://www.numdam.org/item?id=AFST_1999_6_8_2_219_0>

(C) Université Paul Sabatier, 1999, tous droits réservés.

L'accès aux archives de la revue «Annales de la faculté des sciences de Toulouse » (http://picard.ups-tlse.fr/ annales/) implique l'accord avec les conditions générales d'utilisation (http://www.numdam.org/conditions). Toute utilisation commerciale ou impression systématique est constitutive d'une infraction pénale. Toute copie ou impression de ce fichier doit contenir la présente mention de copyright.

\section{Numdam}

Article numérisé dans le cadre du programme

Numérisation de documents anciens mathématiques

http://www.numdam.org/ 


\title{
The defect of weak approximation for homogeneous spaces ${ }^{(*)}$
}

\author{
MikhaIL BorovoI ${ }^{(1)}$
}

RÉSUMÉ. - Soit $X=H \backslash G$ un espace homogène défini sur un corps de nombres $k$, où $G$ est un $k$-groupe linéaire connexe qui satisfait certaines conditions, et où $H$ est un $k$-sous-groupe connexe de $G$. Pour un ensemble fini $S$ de places de $k$, on définit un groupe abélien fini $A_{S}(X)$ qui est le défaut de l'approximation faible pour $X$ par rapport de $S$, et on décrit $A_{S}(X)$ en termes du groupe de Brauer de $X$.

\begin{abstract}
Let $X=H \backslash G$ be a homogeneous space defined over a number field $k$, where $G$ is a connected linear $k$-group satisfying certain conditions, and $H$ is a connected $k$-subgroup of $G$. For a finite set $S$ of places of $k$ we define a finite abelian group $A_{S}(X)$ which is the defect of weak approximation for $X$ with respect to $S$. We describe $A_{S}(X)$ in terms of the Brauer group of $X$.
\end{abstract}

\section{Introduction}

Let $k$ be an algebraic number field, $\mathcal{V}$ the set of places of $k$, and $S \subset \mathcal{V}$ a finite subset. Let $X$ be an algebraic variety over $k$ such that $X(k) \neq \varnothing$. We

(*) Reçu le 23 juin 1998, accepté le 10 novembre 1998

(1) Partially supported by the US-Israel Binational Science Foundation and by the Hermann Minkowski Center for Geometry.

Raymond and Beverly Sackler School of Mathematical Sciences, Tel Aviv University, 69978 Tel Aviv, Israel.

E-mail address: borovoi@math.tau.ac.il 
say that $X$ satisfies the condition of weak approximation with respect to $S$ if

$$
X(k) \text { is dense in } \prod_{v \in S} X\left(k_{v}\right),
$$

$k_{v}$ denoting the completion of $k$ at $v$. We say that $X$ satisfies the condition of weak approximation if

$$
X \text { satisfies }\left(W A_{S}\right) \text { for all finite } S \text {. }
$$

This is the same as to require that $X(k)$ is dense in $\prod_{v \in \mathcal{V}} X\left(k_{v}\right)$.

Let $k_{S}=\prod_{v \in S} k_{v}$; then $X\left(k_{S}\right)=\prod_{v \in S} X\left(k_{v}\right)$. Let $X(k) \hat{S}$ denote the closure of $X(k)$ in $X\left(k_{S}\right)$, and let $X(k)_{\mathcal{V}}$ denote the closure of $X(k)$ in $\prod_{v \in \mathcal{V}} X\left(k_{v}\right)$. Clearly, $\left(W A_{S}\right)$ holds for $X$ if and only if $X(k)_{S}=X\left(k_{S}\right)$, and $(W A)$ holds for $X$ if and only if $X(k) \hat{\mathcal{V}}=\prod_{v \in \mathcal{V}} X\left(k_{v}\right)$.

Let $T$ be an algebraic $k$-torus. Set $A(T)=\left(\prod_{v \in \mathcal{V}} T\left(k_{v}\right)\right) / T(k) \widehat{\mathcal{V}}$. The abelian group $A(T)$ is the defect of weak approximation for $T$; in other words, it is the measure of failure of (WA) for $T$. In particular, $T$ satisfies (WA) if and only if $A(T)=0$. The group $A(T)$ was studied by Voskresenskil [Vo1], [Vo2]. He showed that $A(T)$ is finite, and related it to a certain group $H^{1}(k, \operatorname{Pic}(\bar{V}(T)))$. As Sansuc later showed, $A(T)$ can be computed in terms of the Brauer group of $T$, cf. [Sa], 8.12.

For any connected linear $k$-group $G$ one can define the set $A(G)=$ $\left(\prod_{v \in \mathcal{V}} G\left(k_{v}\right)\right) / G(k)_{\mathcal{V}}$. As above, $A(G)$ is the defect of weak approximation for $G$. Sansuc ([Sa], 3.3), generalizing Voskresenskii's results, proved that the subgroup $G(k)_{\mathcal{V}}$ is normal and that the quotient group $A(G)$ is finite and abelian. He showed that it is possible to compute $A(G)$ in terms of the Brauer group of $G$, cf. [Sa], 8.12.

In this paper we consider the case of a homogeneous space. Let $X=$ $H \backslash G$, where $G$ is a connected linear $k$-group and $H \subset G$ is a connected $k$-subgroup. We suppose that $A(G)=0$ and $\amalg(G)=0$, where $\amalg(G)$ is the Tate-Shafarevich group of $G$. These conditions hold for example when $G$ is a simply connected group, or an adjoint group, or an absolutely simple group, or if $G$ splits over a cyclic extension, cf. [Sa], 5.3. For such $X$ we construct a certain finite abelian group $A_{S}(X)$ which is the defect of $\left(W A_{S}\right)$ for $X$. We construct $A_{S}(X)$ in terms of $H$ and $G$, but then we compute $A_{S}(X)$ in terms of the Brauer group of $X$. We obtain a formula for $A_{S}(X)$ in terms of the Brauer group of $X$, similar to Sansuc's formula ([Sa], 8.12) for $A(G)$ in terms of the Brauer group of $G$ for a connected $k$-group $G$. 
The plan of the paper is as follows. In Section 1 we state the main results. In Section 2 we restate the problem of weak approximation for homogeneous spaces in terms of Galois cohomology. In Section 3 we prove a theorem describing $A_{S}(H \backslash G)$ in terms of $H$ and $G$. In Section 4 we prove a theorem describing $A_{S}(H \backslash G)$ in terms of the Brauer group of $H \backslash G$.

The results of this paper were announced in [Bo1]. The case of a homogeneous space $H \backslash G$ of a simply connected group $G$ was treated in [Bo2].

\section{Acknowledgements.}

This paper was partly written when I was staying at SFB 343 "Diskrete Strukturen in der Mathematik" at Bielefeld University. I am grateful to SFB 343 for hospitality, support, and excellent working conditions.

The paper is inspired by Sansuc's paper [Sa]. The computation of $A_{S}(H \backslash G)$ in terms of $H$ and $G$ is based on results of Kottwitz [Ko1], [Ko2] in the more functorial version of [Bo3].

I am grateful to Boris Kunyavskii for valuable remarks.

\section{Notation}

$k$ is a field of characteristic $0, \bar{k}$ is an algebraic closure of $k, \Gamma=\operatorname{Gal}(\bar{k} / k)$. When $k$ is a number field, then $\mathcal{V}, \mathcal{V}_{\infty}, \mathcal{V}_{f}$ are the set of all places of $k$, of infinite places, of finite places, resp. For $v \in \mathcal{V}, k_{v}$ denotes the completion of $k$ at $v$. For a finite subset $S \subset \mathcal{V}$ we set $k_{S}=\prod_{v \in S} k_{v}$. We write $\mathbf{A}$ for the adèle ring of $k$. For a connected $k$-group $G$ we set

$$
H^{1}\left(k_{S}, G\right)=\prod_{v \in S} H^{1}\left(k_{v}, G\right), \quad H^{1}(\mathbf{A}, G)=\bigoplus_{v \in \mathcal{V}} H^{1}\left(k_{v}, G\right),
$$

where $\bigoplus$ denotes the subset of the direct product consisting of families $\left(\xi_{v}\right)$ for which $\xi_{v}=1$ for all but a finite number of places $v$.

Let $G$ be a connected linear group over a field $F$. Then:

$G^{\mathrm{u}}$ is the unipotent radical of $G$.

$G^{\mathrm{red}}=G / G^{\mathrm{u}} ;$ it is a reductive group.

$G^{\text {ss }}=\left(G^{\text {red }}\right)^{\text {derived }}$; it is a semisimple group.

$G^{\text {tor }}=G^{\text {red }} / G^{\text {ss }}$, it is a torus.

$G^{\text {sssc }}$ denotes the universal covering of $G^{\text {ss }}$; it is a semisimple simply connected group.

$\rho: G^{\text {sssc }} \rightarrow G^{\text {ss }} \rightarrow G^{\text {red }}$ is the composition map.

Let $A$ be a finite abelian group, then $A^{D}$ denotes the dual group for $A$, $A^{D}=\operatorname{Hom}(A, \mathbf{Q} / \mathbf{Z})$. 


\section{Main results}

1.1. Let $k$ be a field of characteristic 0 . Set $\Gamma=\operatorname{Gal}(\bar{k} / k)$. Let $G$ be a connected linear $k$-group. We refer to [Bo3], [Bo1] for the definition of the algebraic fundamental group $\pi_{1}(G)$ (see also 2.1 below). It is a finitely generated abelian group endowed with a $\Gamma$-action. The $\Gamma$-module $\pi_{1}(G)$ depends on $G$ functorially.

Set $B(G)=\left(\pi_{1}(G)_{\Gamma}\right)_{\text {tors }}$, the torsion subgroup of the group of $\Gamma$-coinvariants of $\pi_{1}(G)$. The finite abelian group $B(G)$ depends on $G$ functorially. Kottwitz [Kol], (2.4.1) proved that $B(G)=\operatorname{Pic}(G)^{D}$, the dual group for the Picard group $\operatorname{Pic}(G)$ of $G$.

1.2. Let $k$ be a number field and $S$ a finite set of places of $k$. Let $G$ be a connected linear $k$-group and $H \subset G$ a connected $k$-subgroup. Set $X=H \backslash G$. We are going to construct a finite abelian group $C_{S}(H, G)$ which is the defect of $\left(W A_{S}\right)$ for $X$.

The embedding $H \hookrightarrow G$ defines homomorphisms $\pi_{1}(H) \rightarrow \pi_{1}(G)$ and $B(H) \rightarrow B(G)$. Set $B(H, G)=\operatorname{ker}[B(H) \rightarrow B(G)]$, it is a finite abelian group.

Let $v$ be a place of $k$. Set $\Gamma_{v}=\operatorname{Gal}\left(\bar{k}_{v} / k_{v}\right)$. Fix an embedding $\bar{k} \hookrightarrow \bar{k}_{v}$; then we can regard $\Gamma_{v}$ as a subgroup of $\Gamma$. Set $B_{v}(H, G)=B\left(H_{k_{v}}, G_{k_{v}}\right)$. We obtain a homomorphism $\lambda_{v}: B_{v}(H, G) \rightarrow B(H, G)$. Set $B^{S}(H, G)=$ $\left\langle\lambda_{v}\left(B_{v}(H, G)\right)\right\rangle_{v \notin S}$, the subgroup of $B(H, G)$ generated by the groups $\lambda_{v}\left(B_{v}(H, G)\right)$ for $v \notin S$. Set $B^{\prime}(H, G)=B^{\varnothing}(H, G)$. Then $B^{S}(H, G) \subset$ $B^{\prime}(H, G)$. We set $C_{S}(H, G)=B^{\prime}(H, G) / B^{S}(H, G)$.

1.3. THEOREM. - Let $G, H$ and $X$ be as in 1.2. Suppose that $A(G)=0$ and $\amalg(G)=0$. Then there exists a canonical surjective map $X\left(k_{S}\right) \rightarrow$ $C_{S}(H, G)$ with kernel $X(k) \widehat{S}$.

We see that the condition $\left(W A_{S}\right)$ for $X$ is equivalent to the condition $C_{S}(H, G)=0$. We set $A_{S}(X)=C_{S}(H, G)$ and say that $A_{S}(X)$ is the defect of $\left(W A_{S}\right)$ for $X$.

Theorem 1.3 will be proved in Section 3.

1.4. Set $B^{\omega}(H, G)=\bigcap_{S} B^{S}(H, G)$, where $S$ runs over all the finite subsets of $\mathcal{V}$. We set $C_{\omega}(H, G)=B^{\prime}(H, G) / B^{\omega}(H, G)$. It is clear that $C_{\omega}(H, G)=0$ if and only if $C_{S}(H, G)=0$ for all finite $S \subset \mathcal{V}$.

1.5. Corollary. - $H \backslash G$ satisfies $(W A)$ if and only if $C_{\omega}(H, G)=0$. 
Proof. - Indeed, $C_{\omega}(H, G)=0$ if and only if $C_{S}(H, G)=0$ for all finite $S$. By Theorem 1.3 $C_{S}(H, G)=0$ for all $S$ if and only if $\left(W A_{S}\right)$ holds for $H \backslash G$ for all $S$. Thus $C_{\omega}(H, G)=0$ if and only if $(W A)$ holds for $H \backslash G$.

1.6. Corollary. - Let $L / k$ be a finite Galois extension of $k$ in $\bar{k}$ such that $\operatorname{Gal}(\bar{k} / L)$ acts trivially on $\pi_{1}(H)$ and $\pi_{1}(G)$. Let $S_{0} \subset \mathcal{V}$ be the finite set of places (nonarchimedean, ramified in $L$ ) with noncyclic decomposition groups in $\operatorname{Gal}(L / k)$. Then $C_{S}(H, G)=C_{S \cap S_{0}}(H, G)$. In particular, if $S \cap$ $S_{0}=\varnothing$ then $H \backslash G$ satisfies $\left(W A_{S}\right)$.

Proof. - Let $v \in \mathcal{V}$, and let $w$ be a place of $L$ over $v$. Let $D_{w}$ denote the decomposition group of $w$ in $\mathrm{Gal}(L / k)$. It is easy to show that the subgroup $\lambda_{v}\left(B_{v}(H, G)\right) \subset B(H, G)$ depends only on the conjugacy class of $D_{w}$ in $\operatorname{Gal}(L / k)$. If $D_{w}$ is cyclic and $v \in S$, then, by Chebotarev's density theorem, there exists a place $v^{\prime} \notin S$ such that $\lambda_{v^{\prime}}\left(B_{v^{\prime}}(H, G)\right)=\lambda_{v}\left(B_{v}(H, G)\right)$, and so $B^{S \backslash\{v\}}(H, G)=B^{S}(H, G)$. Thus $B^{S}(H, G)=B^{S \cap S_{0}}(H, G)$ and $C_{S}(H, G)=C_{S \cap S_{0}}(H, G)$. In particular, if $S \cap S_{0}=\varnothing$, then $C_{S}(H, G)=$ $C_{\varnothing}(H, G)=0$, and $H \backslash G$ satisfies $\left(W A_{S}\right)$.

1.7. Corollary (Real approximation). - Let $G, H$ be as in Theorem 1.3. If $S \subset \mathcal{V}_{\infty}$ then $H \backslash G$ satisfies $\left(W A_{S}\right)$.

Indeed, all the archimedean places have cyclic decomposition groups, hence $S \cap S_{0}=\varnothing$, and $H \backslash G$ satisfies $\left(W A_{S}\right)$.

1.8. For completeness we state here a result from [Bo2]. Let $T$ be a $k$ torus. Set $\mathrm{\Psi}_{S}(T)=\operatorname{coker}\left[H^{1}(k, T) \rightarrow H^{1}\left(k_{S}, T\right)\right]$. For a connected $k$-group $H$, let $H^{\text {tor }}$ denote the biggest quotient torus of $H$, see Notation.

1.9. Proposition. - Let $G, H$ be as in 1.2. Assume that $G$ is simply connected. Then $C_{S}(H, G)=\mathrm{Ч}_{S}\left(H^{\text {tor }}\right)$.

Proof. - See [Bo2].

1.10. Let $X$ be a $k$-variety. We denote by $\operatorname{Br}(X)$ the cohomological Brauer group of $X$. Consider the morphisms $X_{\bar{k}} \rightarrow X \rightarrow \operatorname{Spec} k$ and the induced homomorphisms $\operatorname{Br}(k) \stackrel{\alpha}{\longrightarrow} \operatorname{Br}(X) \stackrel{\beta}{\longrightarrow} \operatorname{Br}\left(X_{\bar{k}}\right)$. Set $\mathrm{Br}_{1}(X)=\operatorname{ker} \beta$, $\mathrm{Br}_{a}(X)=\mathrm{Br}_{1}(X) / \mathrm{im} \alpha$.

Let $k$ be a number field, $S$ a finite set of places of $k$. Set $\mathrm{E}_{S}(X)=$ $\operatorname{ker}\left[\operatorname{Br}_{a}(X) \rightarrow \prod_{v \notin S} \operatorname{Br}_{a}\left(X_{k_{v}}\right)\right], \mathrm{B}(X)=\mathrm{b}_{\varnothing}(X), \mathrm{b}_{\omega}(X)=\bigcup_{S} \mathrm{5}_{S}(X)$.

1.11. TheOREM. - Let $G, H, X$ be as in Theorem 1.3. Then $A_{S}(X)=$ $\left(\mathrm{B}_{S}(X) / \mathrm{B}(X)\right)^{D}$. 
Theorem 1.11 will be proved in Section 4 . This theorem shows that the group $A_{S}(X)=C_{S}(H, G)$ does not depend on the representation of $X$ in the form $X=H \backslash G$.

\subsection{Corollary. - Set}

$A(X)=C_{\omega}(H, G)$, then $A(X)=\left(\mathrm{B}_{\omega}(X) / \mathrm{B}(X)\right)^{D}$.

Note that the formula of Corollary 1.12 is similar to Sansuc's formula $A(G)=\left(\mathrm{B}_{\omega}(G) / \mathrm{b}(G)\right)^{D}([\mathrm{Sa}], 8.12)$ for the defect of weak approximation $A(G)$ for a connected $k$-group $G$.

\section{Orbits}

2.1. Let $k, S, G, H, X$ be as in 1.2 , and assume that $A(G)=0$. The group $G$ acts on $X$ on the right. Let $\mathcal{O}(X, G, k)$ denote the set of orbits of the group $G(k)$ in $X(k)$. Let $\mathcal{O}\left(X, G, k_{S}\right)$ denote the set of orbits of $G\left(k_{S}\right)$ in $X\left(k_{S}\right)$. We have a canonical map $i_{S}: \mathcal{O}(X, G, k) \rightarrow \mathcal{O}\left(X, G, k_{S}\right)$ induced by the embedding $X(k) \rightarrow X\left(k_{S}\right)$.

Let $v \in \mathcal{V}, x_{v} \in X\left(k_{v}\right)$, then the morphism $G_{k_{v}} \rightarrow X_{k_{v}}, g \mapsto x_{v} \cdot g$, is smooth. It follows that the map $G\left(k_{v}\right) \rightarrow X\left(k_{v}\right), g \mapsto x_{v} \cdot g$, is open. Now let $x_{S} \in X\left(k_{S}\right)$. We see that the map $G\left(k_{S}\right) \rightarrow X\left(k_{S}\right), g \mapsto x_{S} \cdot g$, is open. Hence the orbit $x_{S} \cdot G\left(k_{S}\right)$ is an open subset in $X\left(k_{S}\right)$. Since all the orbits are open, we conclude that every orbit of $G\left(k_{S}\right)$ in $X\left(k_{S}\right)$ is open and closed.

By assumption $(W A)$ holds for $G$. Hence $\left(W A_{S}\right)$ holds for $G$, i.e. $G(k)$ is dense in $G\left(k_{S}\right)$. Let $o_{S}$ be an orbit of $G\left(k_{S}\right)$ in $X\left(k_{S}\right)$. Assume that $o_{S}$ has a $k$-point $x$. Since the map $G\left(k_{S}\right) \rightarrow X\left(k_{S}\right), g \mapsto x \cdot g$, is continuous, the $G(k)$-orbit $x \cdot G(k)$ is dense in the $G\left(k_{S}\right)$-orbit $o_{S}$. Since $o_{S}$ is closed in $X(k)$, we see that the closure of $x \cdot G(k)$ in $X\left(k_{S}\right)$ is $o_{S}$. We conclude that the closure $X(k) \widehat{S}$ of $X(k)$ in $X\left(k_{S}\right)$ is $\bigcup i_{S}(o)$ where $o$ runs over $\mathcal{O}(X, G, k)$.

\subsection{Set}

$$
K=\operatorname{ker}\left[H^{1}(k, H) \rightarrow H^{1}(k, G)\right], \quad K_{S}=\operatorname{ker}\left[H^{1}\left(k_{S}, H\right) \rightarrow H^{1}\left(k_{S}, G\right)\right] .
$$

The Galois cohomology exact sequences associated with the subgroup $H$ of the group $G$ (cf. [Se], I-5.4, Prop. 36) yield identifications $\mathcal{O}(X, G, k)=K$, $\mathcal{O}\left(X, G, k_{S}\right)=K_{S}$. With these identifications the map $i_{S}: \mathcal{O}(X, G, k) \rightarrow$ $\mathcal{O}\left(X, G, k_{S}\right)$ becomes the restriction to $K$ of the localization map $\operatorname{loc}_{S}: H^{1}(k, H) \rightarrow H^{1}\left(k_{S}, H\right)$.

2.3. We wish to construct an exact sequence $K \stackrel{\text { locs }_{S}}{\longrightarrow} K_{S} \rightarrow C_{S}(H, G) \rightarrow$ 0 . This will give us a surjective map $\mathcal{O}\left(X, G, k_{S}\right) \rightarrow C_{S}(H, G)$ with kernel 
$i_{S}(\mathcal{O}(X, G, k))$, see 2.2. This in turn will give us a surjective continuous map $X\left(k_{S}\right) \rightarrow C_{S}(H, G)$ with kernel $X(k) \widehat{S}$, see 2.1. This will prove Theorem 1.3.

\section{The defect of weak approximation}

In this section we prove Theorem 1.3.

3.1. We recall the definition of the algebraic fundamental group $\pi_{1}(G)$. Let $G$ be a connected linear group over a field $k$ of characteristic 0 . Let $T$ be a maximal torus of the reductive group $G^{\text {red }}$ (see Notation). Consider the composed homomorphism $\rho: G^{\text {sssc }} \rightarrow G^{\text {ss }} \rightarrow G^{\text {red }}$ and set $T^{\text {(sssc) }}=$ $\rho^{-1}(T) \subset G^{\text {sssc }}$. Set

$$
\pi_{1}(G)=\mathrm{X}_{*}(T) / \rho_{*} \mathrm{X}_{*}\left(T^{(\text {sssc })}\right)
$$

where $\mathrm{X}_{*}$ denotes the cocharacter group over $\bar{k}$. Then $\pi_{1}(G)$ does not depend on the choice of $T$. It is a finitely generated abelian group with a $\Gamma$-action, where $\Gamma=\operatorname{Gal}(\bar{k} / k)$. For details see [Bo3], Ch. 1 .

We have defined $B(G)$ by $B(G)=\left(\pi_{1}(G)_{\Gamma}\right)_{\text {tors }}$. Both $\pi_{1}(G)$ and $B(G)$ depend functorially on $G$.

3.2. PROPOSITION. - There is a canonical functorial isomorphism $B(G) \simeq \operatorname{Pic}(G)^{D}$.

Proof. - This result is essentially due to Kottwitz. Kottwitz [Ko1], 2.4.6 constructs an isomorphism $\pi_{0}\left(Z(\hat{G})^{\Gamma}\right) \simeq \operatorname{Pic}(G)$, where $Z(\hat{G})$ is the center of a connected Langlands dual group $\hat{G}$ for $G$. Let $\mathrm{X}^{*}(Z(\hat{G}))$ denote the character group $\operatorname{Hom}\left(Z(\hat{G}), \mathbf{G}_{m, \mathbf{C}}\right)$ of the algebraic $\mathbf{C}$-group of multiplicative type $Z(\hat{G})$. The isomorphism $\mathrm{X}^{*}(Z(\hat{G})) \simeq \pi_{1}(G)$ (cf. [Bo3], 1.10) yields isomorphisms $\mathrm{X}^{*}\left(Z(\hat{G})^{\Gamma}\right) \simeq \pi_{1}(G)_{\Gamma}$ and $\pi_{0}\left(Z(\hat{G})^{\Gamma}\right)^{D} \simeq\left(\pi_{1}(G)_{\Gamma}\right)_{\text {tors }}=B(G)$, whence $B(G) \simeq \operatorname{Pic}(G)^{D}$.

A homomorphism of connected reductive groups $\phi: G_{1} \rightarrow G_{2}$ is called normal if $\phi\left(G_{1}\right)$ is normal in $G_{2}$. A connected Langlands dual group is functorial only with respect to normal homomorphisms, and therefore Kottwitz's $\pi_{0}\left(Z(\hat{G})^{\Gamma}\right)$ is functorial only with respect to normal homomorphisms. However our groups $\pi_{1}(G)$ and $B(G)$ are functorial with respect to all homomorphisms of connected linear groups, and using Lemma 2.4.5 of Kottwitz [Ko1] one can easily show that the isomorphism $B(G) \simeq \operatorname{Pic}(G)^{D}$ is functorial with respect to all homomorphisms.

3.3. Let $k$ be a number field, and let $G$ be a connected linear $k$-group. Set $B_{v}(G)=B\left(G_{k_{v}}\right)$ for $v \in \mathcal{V}$. We have a homomorphism $\lambda_{v}: B_{v}(G) \rightarrow B(G)$, 
which corresponds to the corestriction homomorphism $\left(\pi(G)_{\Gamma_{v}}\right)_{\text {tors }} \rightarrow$ $\left(\pi_{1}(G)_{\Gamma}\right)_{\text {tors }}$, where $\Gamma_{v}$ is a decomposition group.

3.4. Let $F$ be a local field of characteristic 0 . Let $G$ be a connected $F$ group. There is a functorial map $\beta_{F}: H^{1}(F, G) \rightarrow B(G)$, defined by Kottwitz [Ko2], 1.2, see also [Bo3], 3.10 and 4.1(i). The definition of [Bo3] shows that $\beta_{F}$ is functorial with respect to all homomorphisms, not only normal. The $\operatorname{map} \beta_{F}$ is surjective; if $F$ is nonarchimedean, then $\beta_{F}$ is bijective, see [Ko2], 1.2 .

Let $k$ be a number field, $v \in \mathcal{V}$, and let $G$ be a connected $k$-group. We have a map $\beta_{v}=\beta_{k_{v}}: H^{1}\left(k_{v}, G\right) \rightarrow B_{v}(G)$. The map $\beta_{v}$ is surjective. If $v \in \mathcal{V}_{f}$ then $\beta_{v}$ is bijective.

Set

$$
\mu_{v}=\lambda_{v} \circ \beta_{v}: H^{1}\left(k_{v}, G\right) \rightarrow B_{v}(G) \rightarrow B(G) .
$$

Since $\beta_{v}$ is surjective, we have $\operatorname{im} \mu_{v}=\operatorname{im} \lambda_{v}$.

Consider the localization map

$$
\text { loc: } H^{1}(k, G) \rightarrow H^{1}(\mathbf{A}, G)=\bigoplus H^{1}\left(k_{v}, G\right)
$$

(we use the assumption that $G$ is connected). We define a map $\mu=\sum \mu_{v}$ : $H^{1}(\mathbf{A}, G) \rightarrow B(G)$. This map is functorial in $G$.

3.5. Proposition (Kottwitz $[\mathrm{Ko} 2]) .-\operatorname{ker} \mu=\operatorname{im} \operatorname{loc}\left[H^{1}(k, G) \rightarrow\right.$ $\left.H^{1}(\mathbf{A}, G)\right]$.

Proof. - See [Ko2], 2.5 and 2.6. See also [Bo3], 5.16.

3.6. Let $k$ be a number field. Let $H$ be a connected $k$-subgroup of a connected linear $k$-group $G$. Consider the sets

$$
K=\operatorname{ker}\left[H^{1}(k, H) \rightarrow H^{1}(k, G)\right], \quad K_{v}=\operatorname{ker}\left[H^{1}\left(k_{v}, H\right) \rightarrow H^{1}\left(k_{v}, G\right)\right] .
$$

We define

$$
K_{S}=\prod_{v \in S} K_{v}, \quad K_{\mathbf{A}}=\bigoplus_{v \in \mathcal{V}} K_{v} .
$$

Let $B(H, G)$ and $B_{v}(H, G)$ be as in 1.2. Since all our constructions are functorial, we can define maps

$$
\begin{gathered}
\beta_{v}: K_{v} \rightarrow B_{v}(H, G), \quad \lambda_{v}: B_{v}(H, G) \rightarrow B(H, G), \quad \text { loc: } K \rightarrow K_{\mathbf{A}}, \\
\mu_{v}=\lambda_{v} \circ \beta_{v}: K_{v} \rightarrow B(H, G), \quad \mu=\sum \mu_{v}: K_{\mathbf{A}} \rightarrow B(H, G) .
\end{gathered}
$$

Here the symbols $\beta_{v}, \lambda_{v}, \mu_{v}$ and $\mu$ are used to denote the restrictions of the maps defined by the same symbols in 3.3 and 3.4. Note that $B_{v}(H, G)$ 
and $B(H, G)$ are abelian groups and $\lambda_{v}: B_{v}(H, G) \rightarrow B(H, G)$ is a homomorphism.

3.7. Proposition. - For $v \in \mathcal{V}_{f}$ the maps $\beta_{v}: K_{v} \rightarrow B_{v}(H, G)$ are bijections.

Proof. - Consider the commutative diagram with exact rows

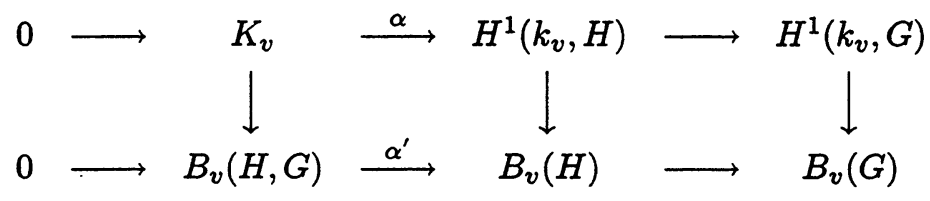

where the maps $\alpha$ and $\alpha^{\prime}$ are injective. The right and the middle vertical arrows are bijective because $v \in \mathcal{V}_{f}$, hence the left vertical arrow is bijective.

3.8. Proposition. - If $\amalg(G)=0$ then

$$
\operatorname{ker}\left[\mu: K_{\mathbf{A}} \rightarrow B(H, G)\right]=\operatorname{im}\left[\text { loc: } K \rightarrow K_{\mathbf{A}}\right] .
$$

Proof. - Consider the commutative diagram with exact rows

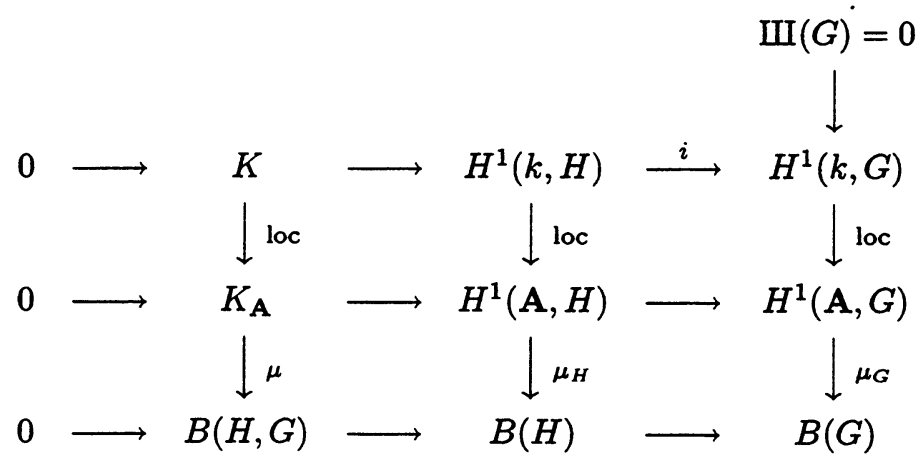

The middle and the right columns of the diagram are exact by Proposition 3.5. By diagram chasing one can prove that the left column is exact.

We write down the diagram chasing. It is easy to see that the composition $K \rightarrow K_{\mathbf{A}} \rightarrow B(H, G)$ is zero. Now let $\xi_{\mathbf{A}} \in K_{\mathbf{A}}, \mu\left(\xi_{\mathbf{A}}\right)=0$. Let $\eta_{\mathbf{A}}$ denote the image of $\xi_{\mathbf{A}}$ in $H^{1}(\mathbf{A}, H)$. Then $\mu_{H}\left(\eta_{\mathbf{A}}\right)=0$, hence $\eta_{\mathbf{A}}=\operatorname{loc}(\eta)$ for some $\eta \in H^{1}(k, H)$. Since $\eta_{\mathbf{A}}$ comes from $K_{\mathbf{A}}$, the image of $\eta_{\mathbf{A}}$ in $H^{1}(\mathbf{A}, G)$ is zero, hence $\operatorname{loc}(i(\eta))=0$. Since $\amalg(G)=0$, we see that $i(\eta)=0$, hence $\eta$ is the image of some $\xi \in K$. Since $\operatorname{loc}(\eta)=\eta_{\mathbf{A}}$, the image of $\operatorname{loc}(\xi)$ in $H^{1}(\mathbf{A}, H)$ is $\eta_{\mathbf{A}}$. The map $K_{\mathbf{A}} \rightarrow H^{1}(\mathbf{A}, H)$ is an embedding, hence $\operatorname{loc}(\xi)=\xi_{\mathbf{A}}$. Thus $\xi_{\mathbf{A}} \in \operatorname{im}\left[\right.$ loc: $\left.K \rightarrow K_{\mathbf{A}}\right]$. 
3.9. Let $B^{\prime}(H, G), B^{S}(H, G)$ and $C_{S}(H, G)$ be as in 1.2. We set $\mu_{S}=$ $\sum \mu_{v}: K_{S} \rightarrow B(H, G), \quad \nu_{S}=\mu_{S} \bmod B^{S}(H, G): K_{S} \rightarrow C_{S}(H, G)$.

3.10. THEOREM. - If $\amalg(G)=0$ then the sequence

$$
K \stackrel{\text { loc }_{S}}{\longrightarrow} K_{S} \stackrel{\nu_{S}}{\longrightarrow} C_{S}(H, G) \rightarrow 0
$$

is exact.

Theorem 1.3 follows from Theorem 3.10, see 2.3.

Proof. - 3.10.1. We prove that $\nu_{S}$ is surjective.

Let $B_{S}(H, G)$ denote the subgroup of $B(H, G)$ generated by the groups $\lambda_{v}\left(B_{v}(H, G)\right)$ for $v \in S$. Then $B^{\prime}(H, G)=B^{S}(H, G)+B_{S}(H, G)$.

Let $v \in \mathcal{V}_{\infty} \cap S$. Using Chebotarev's density theorem, we see that there exists $v^{\prime} \notin S$ such that $\lambda_{v}\left(B_{v}(H, G)\right)=\lambda_{v^{\prime}}\left(B_{v^{\prime}}(H, G)\right)$, and so $B^{S}(H, G)=B^{S \backslash\{v\}}(H, G)$. It follows that $B^{S}(H, G)=B^{S \cap V_{f}}(H, G)$ and $C_{S}(H, G)=C_{S \cap \mathcal{V}_{f}}(H, G)$.

Let $v \in \mathcal{V}_{f}$. By Proposition 3.7 the map $\beta_{v}: K_{v} \rightarrow B_{v}(H, G)$ is bijective. Hence $\mu_{v}\left(K_{v}\right)=\lambda_{v}\left(B_{v}(H, G)\right.$. But $\lambda_{v}\left(B_{v}(H, G)\right)$ is a subgroup of $B(H, G)$ (because $\lambda_{v}$ is a homomorphism), hence $\mu_{v}\left(K_{v}\right)$ is a subgroup of $B(H, G)$.

To prove that $\nu_{S}$ is surjective, we first assume that $S \subset \mathcal{V}_{f}$. Then for any $v \in S, \mu_{v}\left(K_{v}\right)$ is a subgroup of $B(H, G)$, and we see that the image $\mu_{S}\left(K_{S}\right)$ of $K_{S}$ in $B(H, G)$ is a subgroup and equals $B_{S}(H, G)$. But $B^{\prime}(H, G)=$ $B_{S}(H, G)+B^{S}(H, G)$ and

$$
C_{S}(H, G)=B^{\prime}(H, G) / B^{S}(H, G)=\left(B_{S}(H, G)+B^{S}(H, G)\right) / B^{S}(H, G) .
$$

Since $\mu_{S}\left(K_{S}\right)=B_{S}(H, G)$, we see that the map $\nu_{S}: K_{S} \rightarrow C_{S}(H, G)$ is surjective.

Now we do not assume that $S \subset \mathcal{V}_{f}$. The map $K_{S \cap \mathcal{V}_{f}} \rightarrow C_{S \cap \mathcal{V}_{f}}(H, G)=$ $C_{S}(H, G)$ is surjective, hence the map $\nu_{S}: K_{S} \rightarrow C_{S}(H, G)$ is surjective.

3.10.2. We prove that $\nu_{S}\left(\operatorname{loc}_{S}(K)\right)=0$.

Let $x \in K$. Set $x_{S}=\operatorname{loc}_{S}(x) \in K_{S}, x^{S}=\operatorname{loc}^{S}(x) \in K^{S}$, where $K^{S}=$ $\bigoplus_{v \notin S} K_{v}$ and $\operatorname{loc}_{S}, \operatorname{loc}^{S}$ are the localization maps. Let $\mu^{S}: K^{S} \rightarrow B(H, G)$ be the map defined by $\left(x_{v}\right) \mapsto \sum \mu_{v}\left(x_{v}\right)$. By Proposition $3.5 \mu(\operatorname{loc}(x))=0$ for all $x \in K$. Thus $\mu_{S}\left(\operatorname{loc}_{S}(x)\right)+\mu^{S}\left(\operatorname{loc}^{S}(x)\right)=0$. But $\mu^{S}\left(\operatorname{loc}^{S}(x)\right) \in$ $B^{S}(H, G)$. Thus $\nu_{S}\left(\operatorname{loc}_{S}(x)\right)=0$.

3.10.3. We prove that $\operatorname{ker}\left[\nu_{S}: K_{S} \rightarrow C_{S}(H, G)\right] \subset \operatorname{loc}_{S}(K)$. 


\section{The defect of weak approximation for homogeneous spaces}

Consider the map $\mu^{S}: K^{S} \rightarrow B(H, G)$. We prove that $\mu^{S}\left(K^{S}\right)=$ $B^{S}(H, G)$. Using Chebotarev's density theorem we can show that for every $v \in \mathcal{V}_{\infty} \backslash S$ there exists $v^{\prime} \notin S \cup \mathcal{V}_{\infty}$ such that $\lambda_{v}\left(B_{v}(H, G)\right)=$ $\lambda_{v^{\prime}}\left(B_{v^{\prime}}(H, G)\right)$. Thus $B^{S}(H, G)=B^{S \cup \mathcal{V}_{\infty}}(H, G)$. But for $v \notin S \cup \mathcal{V}_{\infty}$ the map $\beta_{v}: K_{v} \rightarrow B_{v}(H, G)$ is bijective (Proposition 3.7), hence $\mu_{v}\left(K_{v}\right)=$ $\lambda_{v}\left(B_{v}(H, G)\right)$. We see that for such $v, \mu_{v}\left(K_{v}\right)$ is a subgroup. Hence

$$
\begin{aligned}
\mu^{S \cup \mathcal{V}_{\infty}}\left(K^{S \cup \mathcal{V}_{\infty}}\right) & =\sum_{v \notin S \cup \mathcal{V}_{\infty}} \mu_{v}\left(K_{v}\right)=\sum_{v \notin S \cup \mathcal{V}_{\infty}} \lambda_{v}\left(B_{v}(H, G)\right) \\
& =B^{S \cup \mathcal{V}_{\infty}}(H, G)=B^{S}(H, G) .
\end{aligned}
$$

But $\mu^{S \cup \mathcal{V}_{\infty}}\left(K^{S \cup \mathcal{V}_{\infty}}\right) \subset \mu^{S}\left(K^{S}\right)$. It follows that $\mu^{S}\left(K^{S}\right)=B^{S}(H, G)$.

Now let $x_{S} \in K_{S}$. Assume that $\nu_{S}\left(x_{S}\right)=0$. Then $\mu_{S}\left(x_{S}\right) \in B^{S}(H, G)$. We have already proved that $B^{S}(H, G)=\mu^{S}\left(K^{S}\right)$, hence $\mu^{S}\left(K^{S}\right)$ is a group and there exists $x^{S} \in K^{S}$ such that $\mu^{S}\left(x^{S}\right)=-\mu_{S}\left(x_{S}\right)$. Set $x_{\mathbf{A}}=$ $\left(x_{S}, x^{S}\right) \in K_{\mathbf{A}}$, then $\mu\left(x_{\mathbf{A}}\right)=0$. By Proposition $3.5 x_{\mathbf{A}}=\operatorname{loc}(x)$ for some $x \in K$. We see that $x_{S}=\operatorname{loc}_{S}(x)$. Thus $\operatorname{ker} \nu_{S} \subset \operatorname{loc}_{S}(K)$. This completes the proof of Theorem 3.10 and Theorem 1.3.

\section{Relation with the Brauer group}

In this section we prove Theorem 1.11.

4.1. Consider the canonical map $G \rightarrow H \backslash G=X$. Set $\operatorname{Br}(X, G)=$ $\operatorname{ker}[\operatorname{Br}(X) \rightarrow \operatorname{Br}(G)], \mathrm{Br}_{a}(X, G)=\operatorname{ker}\left[\operatorname{Br}_{a}(X) \rightarrow \mathrm{Br}_{a}(G)\right]$, where $\mathrm{Br}_{a}(X)$ is defined in 1.10. The torsor $G \rightarrow X$ under $H$ gives rise to the exact sequence

$$
\operatorname{Pic}(G) \rightarrow \operatorname{Pic}(H) \rightarrow \operatorname{Br}(X) \rightarrow \operatorname{Br}(G),
$$

cf. [Sa], (6.10.1). Write $\operatorname{Pic}(G, H)=\operatorname{coker}[\operatorname{Pic}(G) \rightarrow \operatorname{Pic}(H)], \operatorname{Pic}_{1}(G, H)=$ $\operatorname{ker}\left[\operatorname{Pic}(G, H) \rightarrow \operatorname{Pic}\left(G_{\bar{k}}, H_{\bar{k}}\right)\right]$. From the commutative diagram with exact rows

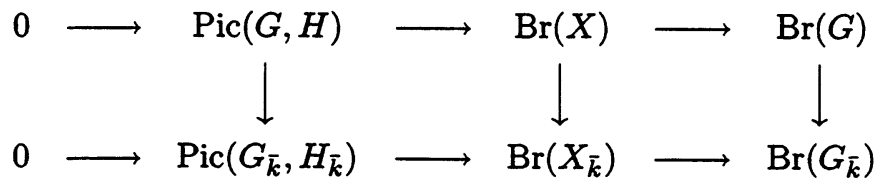

we obtain the exact sequence

$$
0 \rightarrow \operatorname{Pic}_{1}(G, H) \rightarrow \mathrm{Br}_{1}(X) \rightarrow \mathrm{Br}_{1}(G),
$$

where $\mathrm{Br}_{1}$ is defined in 1.10 . 
4.2. We have

$\operatorname{Pic}(G, H)^{D}=\operatorname{coker}[\operatorname{Pic}(G) \rightarrow \operatorname{Pic}(H)]^{D}=\operatorname{ker}[B(H) \rightarrow B(G)]=B(H, G)$.

Set

$$
\begin{array}{r}
B_{1}(H, G)=\operatorname{Pic}_{1}(G, H)^{D}=\operatorname{ker}\left[\operatorname{Pic}(G, H) \rightarrow \operatorname{Pic}\left(G_{\bar{k}}, H_{\bar{k}}\right)\right]^{D} \\
=\operatorname{coker}\left[\operatorname{Pic}\left(G_{\bar{k}}, H_{\bar{k}}\right)^{D} \rightarrow \operatorname{Pic}(G, H)^{D}\right] \\
=\operatorname{coker}\left[B\left(H_{\bar{k}}, G_{\bar{k}}\right) \rightarrow B(H, G)\right] .
\end{array}
$$

We define $B_{1 v}(H, G), B_{1}^{S}(H, G), B_{1}^{\prime}(H, G)$ and $C_{1 S}(H, G)$ like $B_{v}(H, G), \ldots$, $C_{S}(H, G)$ but with $B_{1}(H, G)$ instead of $B(H, G)$, i.e. $B_{1 v}(H, G)=B_{1}\left(H_{k_{v}}\right.$, $\left.G_{k_{v}}\right), B_{1}^{S}(H, G)=\left\langle\lambda_{v}\left(B_{1 v}(H, G)\right)\right\rangle_{v \notin S}, B_{1}^{\prime}(H, G)=B_{1}^{\varnothing}(H, G), C_{1 S}(H, G)=$ $B_{1}^{\prime}(H, G) / B_{1}^{S}(H, G)$.

4.3. Lemma. - $C_{1 S}(H, G)=C_{S}(H, G)$.

Proof. - Let $\bar{\lambda}: B\left(H_{\bar{k}}, G_{\bar{k}}\right) \rightarrow B(H, G)$ be the homomorphism induced by the morphism of pairs $\left(H_{\bar{k}}, G_{\bar{k}}\right) \rightarrow(H, G)$. We have $B\left(H_{\bar{k}}, G_{\bar{k}}\right)=B\left(H_{\bar{k}_{v}}\right.$, $G_{\bar{k}_{v}}$ ). From the commutative diagram of pairs

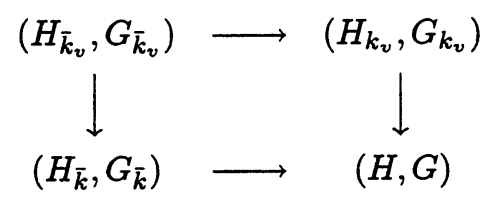

we obtain the commutative diagram

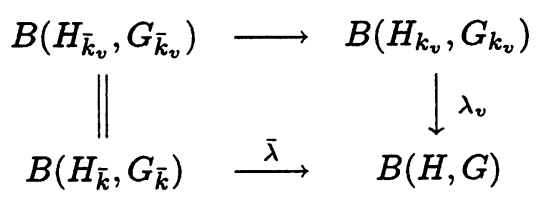

We see that $\operatorname{im}\left[\lambda_{v}: B_{v}(H, G) \rightarrow B(H, G)\right] \supset \operatorname{im} \bar{\lambda}$. Hence $B^{S}(H, G) \supset$ $\operatorname{im} \bar{\lambda}$ and $B^{\prime}(H, G) \supset \operatorname{im} \bar{\lambda}$. We have $B_{1}(H, G)=B(H, G) / \operatorname{im} \bar{\lambda}$. Hence

$$
\begin{aligned}
C_{1 S}(H, G) & =B_{1}^{\prime}(H, G) / B_{1}^{S}(H, G)=\left(B^{\prime}(H, G) / \operatorname{im} \bar{\lambda}\right) /\left(B^{S}(H, G) / \operatorname{im} \bar{\lambda}\right) \\
& =B^{\prime}(H, G) / B^{S}(H, G)=C_{S}(H, G) .
\end{aligned}
$$

4.4. Let $X$ be a $k$-variety. A $k$-point $x \in X(k), x: \operatorname{Spec}(k) \rightarrow X$, defines a homomorphism $x^{*}: \mathrm{Br}_{1}(X) \rightarrow \mathrm{Br}_{1}(k)=\operatorname{Br}(k)$. Define $\mathrm{Br}_{x}(X)=$ $\operatorname{ker}\left[x^{*}: \operatorname{Br}_{1}(X) \rightarrow \operatorname{Br}(k)\right]$. There is a structure morphism $s: X \rightarrow \operatorname{Spec}(k)$ defining a homomorphism $s^{*}: \operatorname{Br}(k) \rightarrow \mathrm{Br}_{1}(X)$. We have $x^{*} \circ s^{*}=1_{\mathrm{Br}(k)}$. Using these two homomorphisms we can prove that $\mathrm{Br}_{1}(X)=\mathrm{Br}_{x}(X) \oplus \operatorname{Br}(k)$ and that $\mathrm{Br}_{x}(X) \simeq \mathrm{Br}_{a}(X)$. 
Now let $X=H \backslash G$ as before. We set $\operatorname{Br}_{1}(X, G)=\operatorname{ker}\left[\mathrm{Br}_{1}(X) \rightarrow\right.$ $\left.\mathrm{Br}_{1}(G)\right]$. We have proved in (4.1.1) that $\operatorname{Pic}_{1}(G, H)=\mathrm{Br}_{1}(X, G)$. We have

$$
\mathrm{Br}_{1} X=\mathrm{Br}_{x_{0}}(X) \oplus \mathrm{Br}(k), \quad \mathrm{Br}_{1}(G)=\mathrm{Br}_{e}(X) \oplus \mathrm{Br}(k)
$$

where $x_{0}$ denotes the image in $X(k)$ of the unit element $e \in G(k)$. We have isomorphisms $\mathrm{Br}_{a}(X)=\mathrm{Br}_{x_{0}}(X), \mathrm{Br}_{a}(G)=\mathrm{Br}_{e}(G)$, whence

$$
\begin{aligned}
\operatorname{Pic}_{1}(G, H) & =\operatorname{Br}_{1}(X, G)=\operatorname{ker}\left[\mathrm{Br}_{x_{0}}(X) \oplus \operatorname{Br}(k) \rightarrow \mathrm{Br}_{e}(G) \oplus \operatorname{Br}(k)\right] \\
& =\operatorname{ker}\left[\mathrm{Br}_{x_{0}}(X) \rightarrow \mathrm{Br}_{e}(G)\right] \\
& =\operatorname{ker}\left[\mathrm{Br}_{a}(X) \rightarrow \mathrm{Br}_{a}(G)\right]=\mathrm{Br}_{a}(X, G) .
\end{aligned}
$$

We define the groups $\mathrm{b}_{S}(X, G)$ and $\mathrm{b}(X, G)$ in terms of $\mathrm{Br}_{a}(X, G)$ in the same way as $\mathrm{B}_{S}(X)$ and $\mathrm{B}(X)$ were defined in terms of $\mathrm{Br}_{a}(X)$, i.e. $\mathrm{Б}_{S}(X, G)=\operatorname{ker}\left[\operatorname{Br}_{a}(X, G) \rightarrow \prod_{v \notin S} \operatorname{Br}_{a}\left(X_{k_{v}}, G_{k_{v}}\right)\right], \mathrm{B}(X, G)=\mathrm{Б}_{\varnothing}(X, G)$.

4.5. Proposition. $-C_{S}(H, G)=\left(\mathrm{b}_{S}(X, G) / \mathrm{b}(X, G)\right)^{D}$.

Proof. - We have

$$
B_{1}(H, G)=\operatorname{Pic}_{1}(H, G)^{D}=\operatorname{Br}_{a}(X, G)^{D}, \quad B_{1 v}(H, G)=\mathrm{Br}_{a}\left(X_{k_{v}}, G_{k_{v}}\right)^{D} .
$$

For a homomorphism $\varphi: A \rightarrow B$ of torsion abelian groups we have

$$
\operatorname{im}[\varphi: A \rightarrow B]^{D}=B^{D} /(\operatorname{coker} \varphi)^{D}=B^{D} / \operatorname{ker}\left[\varphi^{D}: B^{D} \rightarrow A^{D}\right] .
$$

In our case take

$$
A=\bigoplus_{v \notin S} B_{1 v}(H, G), \quad B=B_{1}(H, G),
$$

then

$$
A^{D}=\prod_{v \notin S} \mathrm{Br}_{a}\left(X_{k_{v}}, G_{k_{v}}\right), \quad B^{D}=\mathrm{Br}_{a}(X, G)
$$

and so

$$
\begin{aligned}
B_{1}^{S}(H, G)^{D} & =\operatorname{im}\left[\bigoplus_{v \notin S} B_{1 v}(H, G) \rightarrow B_{1}(H, G)\right]^{D} \\
& =\operatorname{im}[A \rightarrow B]^{D}=B^{D} / \operatorname{ker}\left[B^{D} \rightarrow A^{D}\right] \\
& =\left(\operatorname{Br}_{a}(X, G) / \operatorname{ker}\left[\operatorname{Br}_{a}(X, G) \rightarrow \prod_{v \notin S} \operatorname{Br}_{a}\left(X_{k_{v}}, G_{k_{v}}\right)\right]\right) \\
& =\left(\operatorname{Br}_{a}(X, G) / \mathrm{B}_{S}(X, G)\right) .
\end{aligned}
$$

Thus

$$
\begin{gathered}
B_{1}^{S}(H, G)=\left(\mathrm{Br}_{a}(X, G) / \mathrm{Б}_{S}(X, G)\right)^{D} \\
B_{1}^{\prime}(H, G)=B_{1}^{\varnothing}(H, G)=\left(\operatorname{Br}_{a}(X, G) / \mathrm{B}(X, G)\right)^{D} . \\
-231-
\end{gathered}
$$


Using this we obtain

$$
\begin{aligned}
C_{S}(H, G)=C_{1 S}(H, G) & =B_{1}^{\prime}(H, G) / B_{1}^{S}(H, G) \\
& =\left(\operatorname{Br}_{a}(X, G) / \mathrm{b}(X, G)\right)^{D} /\left(\mathrm{Br}_{a}(X, G) / \mathrm{Б}_{S}(X, G)\right)^{D} \\
& =\left(\mathrm{B}_{S}(X, G) / \mathrm{B}(X, G)\right)^{D} .
\end{aligned}
$$

4.6. Proposition. - If $\amalg(G)=0$ and $A(G)=0$, then $\mathrm{B}(G)=0$ and $\mathrm{B}_{S}(G)=0$.

Proof. - By [Sa], 8.14 there exists an exact sequence

$$
0 \rightarrow A(G) \rightarrow \mathrm{B}_{\omega}(G) \rightarrow \mathrm{\amalg}(G) \rightarrow 0 .
$$

Since $A(G)=0$ and $\amalg(G)=0$, we see that $\mathrm{Б}_{\omega}(G)=0$. Since $\mathrm{Б}_{S}(G) \subset$ $\mathrm{b}_{\omega}(G)$, we conclude that $\mathrm{Б}_{S}(G)=0$. Take $S=\varnothing$, we obtain that $\mathrm{\textrm {b }}(G)=0$.

4.7. Lemma. - If $\mathrm{b}_{S}(G)=0$ then $\mathrm{B}_{S}(X, G)=\mathrm{b}_{S}(X)$.

Proof. - Consider the commutative diagram with exact columns

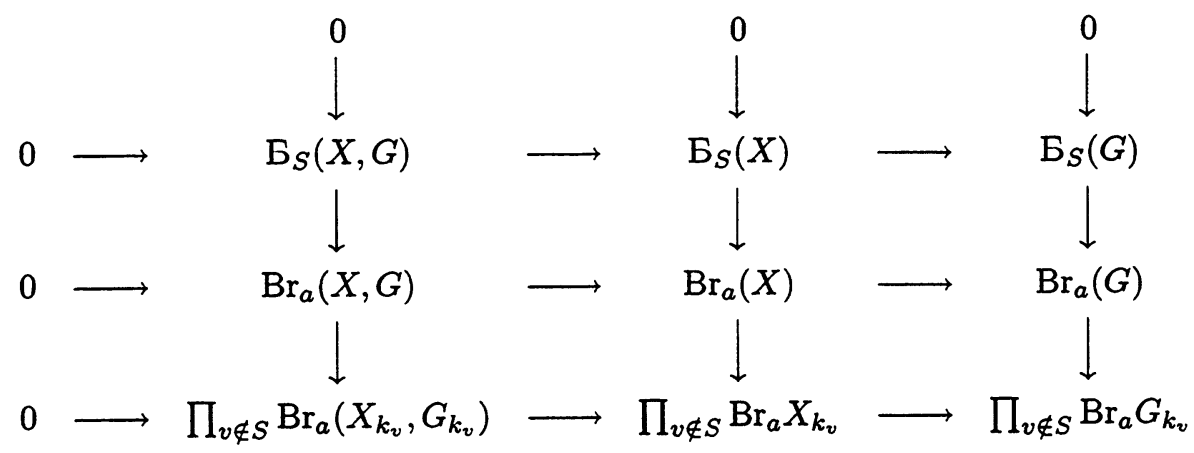

From the fact that the middle row and the lower row are exact, one can easily deduce that the upper row is exact. Since $\mathrm{b}_{S}(G)=0$, we see from the diagram that $\mathrm{b}_{S}(X, G)=\mathrm{B}_{S}(X)$.

4.8. It follows from Proposition 4.6, Lemma 4.7 and Proposition 4.5 that $C_{S}(H, G)=\left(\mathrm{B}_{S}(X) / \mathrm{B}(X)\right)^{D}$. This proves Theorem 1.11. 
The defect of weak approximation for homogeneous spaces

\section{Bibliography}

[Bol] Borovor M., On weak approximation in homogeneous spaces of algebraic groups, Soviet Math. Dokl. 42 (1991), pp. 247-251.

[Bo2] BoROVOI M., On weak approximation in homogeneous spaces of simply connected algebraic groups, Proc. Internat. Conf. "Automorphic Functions and Their Applications, Khabarovsk, June 27 - July 4, 1988" (N. Kuznetsov and V. Bykovsky, eds.), Khabarovsk, 1990, pp. 64-81.

[Bo3] Borovor M., Abelian Galois cohomology of reductive group, Memoirs of Amer. Math. Soc. 132 (1998), no. 626.

[Ko1] KoTTwITz R.E., Stable trace formula: cuspidal tempered terms, Duke Math. J. 51 (1984), pp. 611-650.

[Ko2] KotTwitz R.E., Stable trace formula: elliptic singular terms, Math. Ann. 275 (1986), pp. 365-399.

[Sa] SANSUC J.-J., Groupe de Brauer et arithmétique des groupes algébriques linéaires sur un corps de nombres, Jfür die reine und angew. Math. 327 (1981), pp. 12-80.

[Vo1] Voskresenskir V.E., Birational properties of linear algebraic groups, Izv. Akad. Nauk SSSR 34 (1970), pp. 3-19; English transl. in Math. USSR Izv. 4 (1970), pp. 1-17.

[Vo2] VoskresensKir V.E., Algebraic Groups and their Birational Invariants, Translations of Mathematical Monographs, vol. 179, AMS, Providence, Rhode Island, 1998. 